\title{
Silicon based cell sorting device: Fabrication, characterization and applications
}

\author{
Bivragh Majeed, Chengxun Liu, Erik Sohn, Lut Van Acker, Koen De Wijs, Deniz Sabuncuoglu and Liesbet \\ Lagae \\ IMEC Leuven \\ IMEC, Kapeldreef 75, B3001 Leuven, Belgium. \\ Ph: +32-(0)16-28 7735; Fax: +32-(0)16-28 1097 \\ E-mail: bivragh.majeed@imec.be
}

\begin{abstract}
Cell sorting is an important diagnostic tool used in various aspect of medical prognosis. Numerous cell sorting techniques are currently available on market but they are quite bulky, expensive and at the same time requires very trained operators. In this paper we report on wafer level fabrication technique that will allows for a small form factor device targeting cell sorting. We give detailed overview for the fabrication of our miniaturized cell sorter that is formed with a CMOS compatible process. It used standard fabrication technique in combination with photo-patternable polymer, that has excellent properties for microfluidics applications. The ability to process on wafer level distinguish this from other processes, whose yields are limited to few test samples. The device fabrication includes: processing of micro-heaters, definition of polymer microfluidic channels and collective die-to-wafer bonding of glass substrate onto the polymer channels. We report on the initial characterization of the cell sorting targeting sorting rate and sorting yield. We have achieved sorting rate of 5,000 cells/s and yield of 70\% is obtained in initial investigations.
\end{abstract}

\section{Key words}

Silicon microfabrication, Polymer adhesive, Collective bonding, Cell sorting, Micro-bubbles

\section{Introduction}

In this paper we describe the fabrication process for a microfluidics device targeting cell sorting applications. Currently there are numerous commercial products that are available for cell sorting, including Sony's SH800S Cell Sorter, Bio_Rad S3e $\mathrm{e}^{\mathrm{TM}}$ cell sorter and BD FACSAria sorter to name a few, however they are quite bulky and expensive and at the same time quite complex to operate. Most cell sorting techniques are based on Fluorescence activated cell sorting (FACS). FACS is an important diagnostic tool in microbiology to separate different type of cells at a very high throughput. More detail review about the history and recent update can be found in many references [1], [2]. At imec, for last many years we have been developing a simple and generic on-chip cell sorting system. [3]-[5]. In this paper we report on wafer level fabrication technique that will allows for a small form factor device targeting cell sorting. The aim is to develop a system that will move away from centralized facilities and will be available as a pointof-care tool. It will require small sample volumes and will be more user-friendly than current systems. The basic principle of our device is generation of heat pulse on a heater element that will create a bubble jet flow, enabling the sorting. The device fabrication includes: processing of micro metal heaters, definition of polymer microfluidic channels and collective die-to-wafer bonding of glass substrate onto the polymer channels.

In our previous paper we reported on the fabrication of cell sorter based on Aluminum heaters [6]. However, one of the drawbacks of the this device was poor reliability due to low melting temperature of Al. From our results we estimated that during repeated heating cycles the temperature of heater can reach up to $550^{\circ} \mathrm{C}$ which is close to the $\mathrm{Al}$ melting point. By increasing the number of cycling to millions of cycles, this temperature can reach even higher. At the same time the reliability of device is further reduced due to electro migration due to high current density on the hot spot region [7], [8]. Fig. 1 shows the damage to the Al heater on the hot spot from our first generation cell sorting device. The damage was attributed to above mentioned reasons. Another weak point in our initial scheme was the oxide passivation on top of $\mathrm{Al}$ heater. As shown in Fig 1b, the hot spot is completed disintegrated including the oxide passivation. In repeated firing cycles, a high current passes through the heater resulting in local thermal cycling. There is a larger CTE mismatch between oxide and $\mathrm{Al}$ during this 
thermal cycling and this could also lead to damage shown in the Fig. 1 .

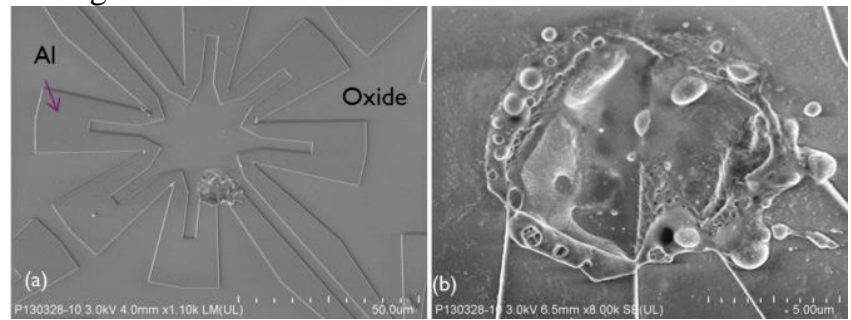

Figure 1(a) the heating element cosnsit of Al heater covered with oxide (b) zoom image of the damaged $\mathrm{Al} /$ oxide

To overcome this issue and increase the reliability of cell sorter device, a different processing scheme was developed. There are two main changes compared to our previous work, first the heaters are made of tungsten and second the passivation stack on top of heater was changed from silicon oxide to silicon nitride and silicon carbide. Although we have used tungsten as a heating element but Tungsten is too resistive to be used a fan-out layer for the wire-bonding layer. For this reason we have to open the passivation and make contact with Aluminum and use this a fan-out layer. However, this possess additional challenges, firstly it increase the total number of step and fabrication complexity, secondly the interconnect layer now becomes the weakest point. The step coverage at this opening is an important parameter to avoid electromigration effects, because $\mathrm{Al}$ can be much thinner specially at the passivation opening. For this reason we have come up with the process flow describe below that reduces the topography around areas of the interconnect The second improvement involves change of passivation layers that will improve the yield by reducing the thermal expansion mismatch. Hewlett Packard has used similar passivation for its inkjet printer as given in reference [9], [10].

The next development discussed in the paper is that of a polymer material. This material is used for the definition of microfluidics channels and used a bonding medium for glass die to silicon bonding. The polymer is a negative tone photo-patternable adhesive. It has excellent resolution, has low curing temperature, has straight patterning profile essential for microfluidics channels and very low or no degassing during the bonding step. It can be used for die to die or die to wafer bonding of Si onto glass [11], [12]. Lastly we report on the initial characterization of the cell sorting targeting sorting rate and sorting yield.

\section{Process flow}

The process start with the deposition of 300nm silicon nitride on a $200 \mathrm{~mm}$ silicon wafer. This $\mathrm{SiN}$ is chosen to reduce the thermal mismatch between heating element and silicon wafer. An etch stop layer of $10 \mathrm{~nm} \mathrm{Al}{ }_{2} \mathrm{O}_{3}$ is deposited before the heater stack of 300nm tungsten is deposited. Photolithography is done to define the heater pattern. Each mask layer is stitched to make one die with a die size of $4.2 \times 2.1 \mathrm{~cm}$. This give us roughly 20 good dies per wafer. A dry etch is done to defined the heater element (Fig. 2a). In the next step, 300nm $\mathrm{SiN}$ passivation is deposited and patterned is defined with lithography for opening of the passivation (Fig. 2b). A dry etch step selective to tungsten is used to open the passivation. The passivation remains on the heating element and only opened in the contact area. A second passivation layer $300 \mathrm{~nm} \mathrm{SiC} \mathrm{layer} \mathrm{is} \mathrm{then} \mathrm{deposited,}$ patterned defined and etched (fig c). The reason for two step etch is to reduce the step height for Al deposition. After passivation opening, $\mathrm{Al}$ is deposited, patterned and dry etched making the connection from heater element to bond pad area (Fig. 2d). Next a 500nm oxide passivation is deposited to protect $\mathrm{Al}$ coming in contact with the liquid during functioning of the device. The passivation is then open at the bond pad as well as on top of the heater element (Fig. 2e). In the next step, a polymer is spin coated and exposed to define the microfluidic channel (Fig. 2f). The polymer is also used as a bonding agent, where a prepunched glass die are bonded onto the device wafer. In the final step, the polymer is cured to finished the bonding and wafer are diced in individual dies for further characterization (Fig 2g). The glass is pre-punched allowing the polymer microfluidics channels connection to the outside testing equipment.
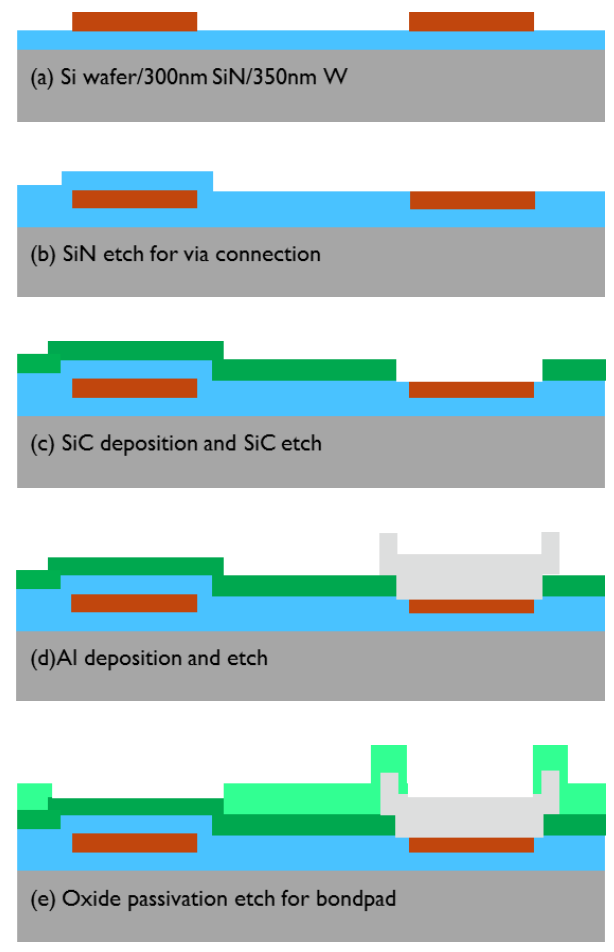

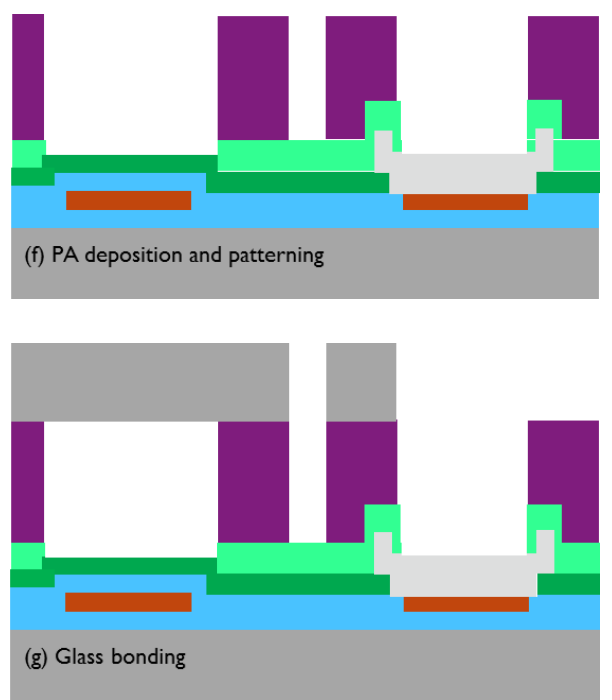

Figure 2: Different step discussed above are schematically shown in figure (a)Tungsten heater definition, (b) SiN etch, (c) $\mathrm{SiC}$ etch, (d) $\mathrm{Al}$ metallization and etch, (e) oxide passivation, (f) polymer adhesive and (g) final bonding to glass chip

\section{Results}

\section{A. Fabrication results:}

In this section, results from different fabrication steps are given. Each main step is given with a cross-section scanning electron microscopy (SEM) image. Fig. 3a shows the cross-section image of the heater element showing the over etch into $\mathrm{SiN}$ and silicon. To overcome this problem, and etch stop layer of $\mathrm{Al}_{2} \mathrm{O}_{3}$ was deposited. During the etch first tungsten is etched with $\mathrm{SF}_{6}$ and $\mathrm{CH}_{3}$ chemistry in a dry etch tool. The etch point is detected with end point trace in the tool and finally $\mathrm{BCl}_{3}$ chemistry is changed to etch remaining $\mathrm{Al}_{2} \mathrm{O}_{3}$ etch stop layer. Fig (b) shows the final result where very good selectivity for Tungsten etch to etch stop layer is visible.

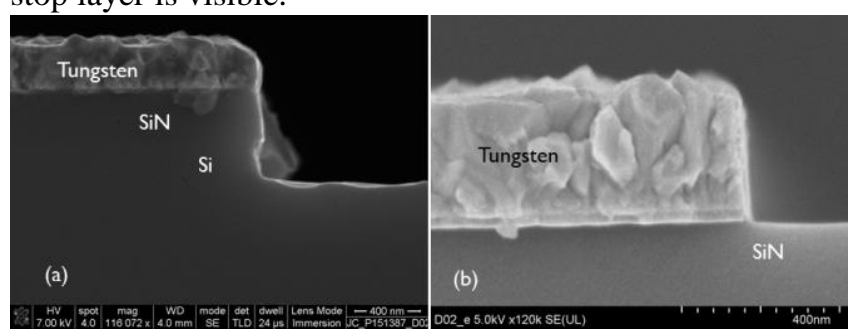

Figure 3 (a): W-etch without the etch stop layer, (b) final etch of heating element.

In the next step, 300nm $\mathrm{SiN}$ passivation is deposited, followed by lithography to define the pattern. There is a 200nm overlap between tungsten and SiN etch. Fig4. (a) shows the cross-sectional image after SiN etch. A dry etch process using $\mathrm{Ar}, \mathrm{O}_{2}, \mathrm{CF}_{4}, \mathrm{CHF}_{3}$ gasses is used. Etch is selective to tungsten. A slight over etch of the $\mathrm{SiN}$ in the overlap area is also shown. Figure 4(b) shows the heater element where the $\mathrm{SiN}$ is still protecting the tungsten heater. A combination of dry and wet etch chemistries are used for the stripping of the resist.

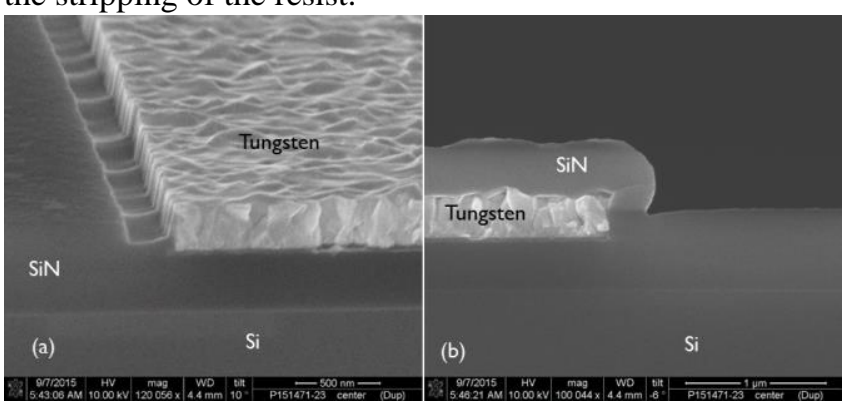

Figure 4 (a) $\mathrm{SiN}$ etch, (b) area where $\mathrm{SiN}$ is not etched

The next step is addition of $300 \mathrm{~nm}$ Silicon carbide to the stack followed by patterning and etching. The etching was done using $\mathrm{Ar} \mathrm{O}_{2}, \mathrm{CF}_{4}, \mathrm{CHF}_{3}$. This etch is also very selective to tungsten and no effect on tungsten was observed (Fig. 5a). The selectivity to $\mathrm{SiN}$ is also very good. There is slight over etch in the region next to tungsten heater but this is due to mask design. Fig. 5 (b) shows the conformal covering over heater element for $\mathrm{SiN}$ and $\mathrm{SiC}$.

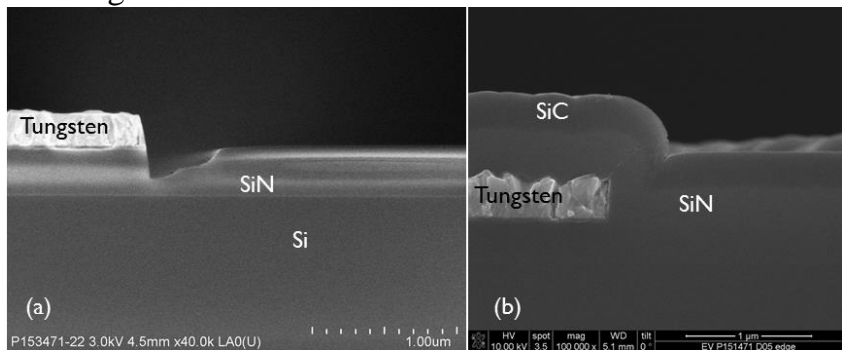

Figure 5: (a) SiC etch landing on $\mathrm{W}$ in interconnect area, (b) heater element covered with $\mathrm{SiN}$ and $\mathrm{SiC}$.

After the passivation opening, a metal stack consisting of $\mathrm{Ti} / \mathrm{TiN} / \mathrm{Al} / \mathrm{TiN}$ is deposited. The first $\mathrm{Ti} / \mathrm{TiN}$ are adhesion and barrier layer while the last TiN is used for electrode inside the channel. The patterned is defined and $\mathrm{Al}$ is etch landing on TiN. Fig. 6 shows four different region of the wafer after the Al etch. Fig. 6a shows the heating element where all the $\mathrm{Al}$ is etched with remaining $\mathrm{SiN}$ and $\mathrm{SiC}$ on top of heater element. Fig. $6 \mathrm{~b}$ shows the Al coverage at the passivation opening. This is the weakest point as $\mathrm{Al}$ could be thinnest at the step coverage. However, in our process we have step of only $300 \mathrm{~nm}$ while Al stack is $800 \mathrm{~nm}$ thick and as shown in figure we have good coverage over this step. This would reduce the failure at this point due to electromigration. Fig. 6c shows the contact between tungsten and $\mathrm{Al}$ at the passivation opening. Al is deformed during cleaving of the sample for SEM analysis but good 
contact was made between the two metals. Fig. 6d shows the $\mathrm{Al}$ etch on the line landing on $\mathrm{SiC}$. There is some over etch on $\mathrm{SiC}$ with this recipe but for the current application this is acceptable. One of the ways to overcome this issue is to use another etch stop layer.

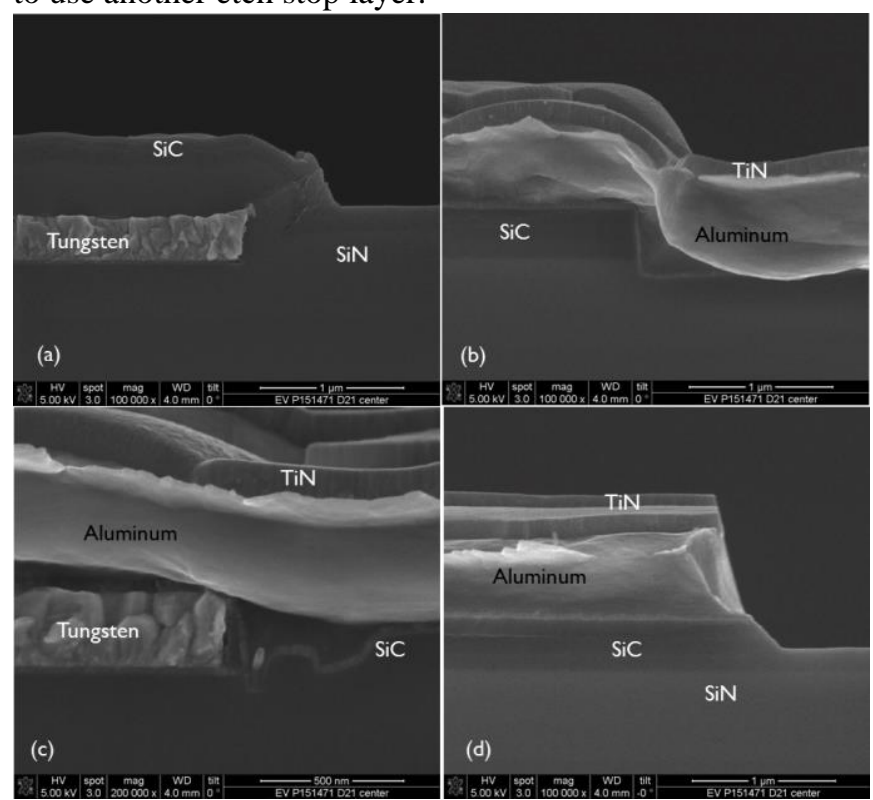

Figure 6 (a-d): four different locations on the die after $\mathrm{Al}$ etch.

In the next step, a top oxide was deposited to protect $\mathrm{Al}$ that otherwise would come in contact with fluid during the testing. Fig. 7 (a-b) shows first the deposited oxide on top of heating element and on Al lines, while Fig. 7(c-d) shows die after the oxide etch. We have used a selective recipe so as not to consume the top SiC layer as shown in Fig 7c. Lastly, in the next etch, the remaining TiN on the bond pad is dry etched and wafers are ready for further processing.

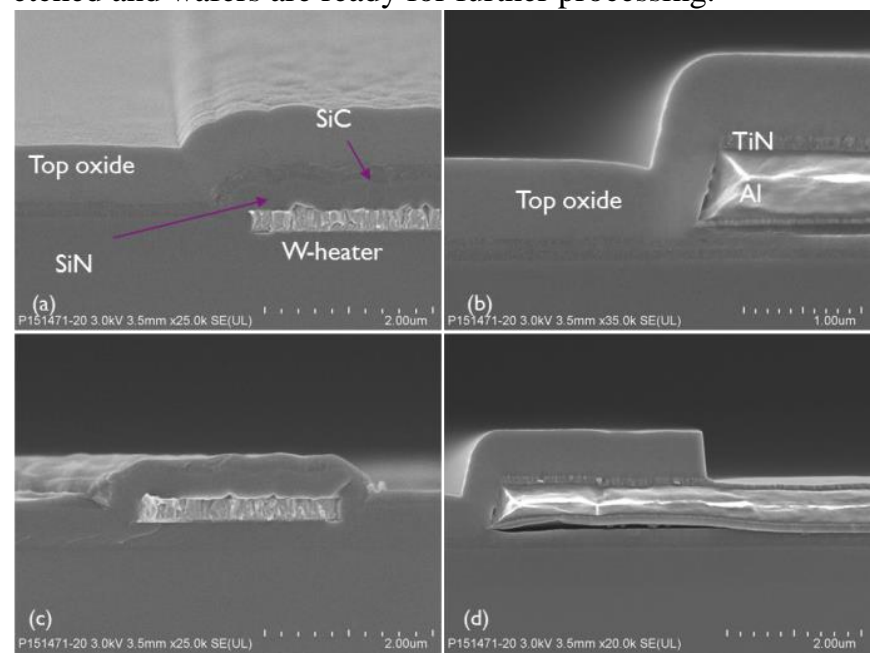

Figure 7 (a-b): After top oxide deposition, (c-d) after oxide etch to open the bond pads

\section{B. Polymer adhesive optimization results:}

In the next part, parallel work done on the development of a polymer layer will be described. The polymer material not only used to define the microfluidic channel but also act as bonding material between silicon and glass. Initial development for the polymer adhesive was presented in our earlier paper [6]. In the current device the minimum channels are $30 \mu \mathrm{m}$ wide and thickness of the polymer is also $30 \mu \mathrm{m}$. There was also design change to allow for multichannel sorting using 5 different channels.

We had developed a process with double coating of material and optimized processed condition to obtain $30 \mu \mathrm{m}$ channel height. In this work we report on a new version of the material that allowed to spin coat the material in one step to obtain the required channel height. Initial we tested the chemical compatibility of the material in our coating equipment. Wafer were spin coated at different speed and coating the thickness was measured by contact profilometer and ellipsometry. The difference between two methods for thickness measurements was less than 260nm. For further studies we only relied on elliospmetry as it allowed measurement of 49 points across the wafer and provided more information on uniformity. Fig. 8 gives the spin speed curve for the polymer adhesive material and shows we can spin coat the layer with a single coat with a target thickness of $30 \mu \mathrm{m}$.

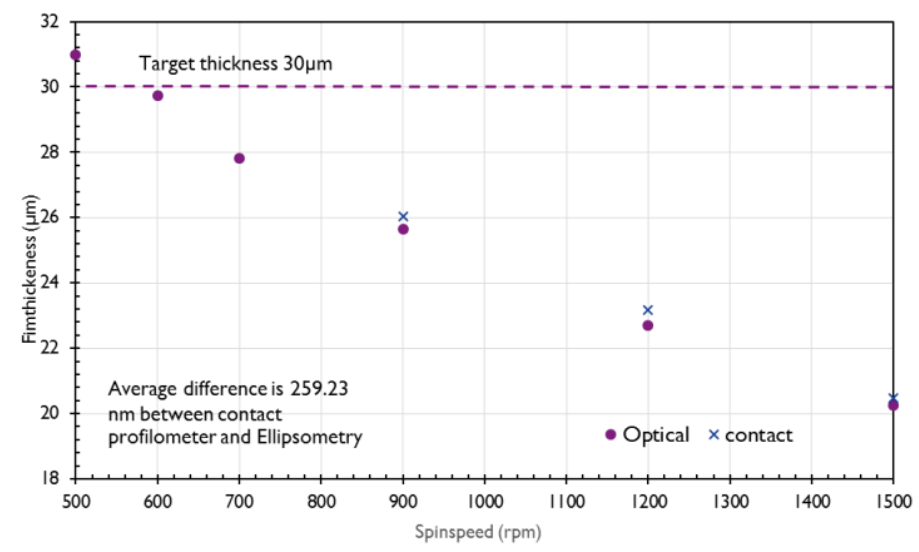

Figure 8: Spin speed curve for polymer adhesive material

After the coating, exposure and post exposure bake conditions were optimized. The exposure was done on an Ultratech AP300 tool. The energy dose was used from 3500 to $5500 \mathrm{~mJ}$ with a step of $500 \mathrm{~mJ}$ After exposure a post exposure bake (PEB) of $110^{\circ} \mathrm{C}$ for $300 \mathrm{sec}$ and two times $60 \mathrm{sec}$ puddle with $\mathrm{TMAH}(2.38 \%)$, was done followed with $60 \mathrm{sec}$ rinse. From the energy does experiment it was concluded that $5500 \mathrm{~mJ}$ energy was needed for the optimal processing of the polymer adhesive. It allowed for straight channel profile that is needed for microfluidics channels and also no residues were observed in the channel. After initial optimization, the process was applied to device wafer as 
shown in Fig. 9. Fig. 9a shows the microfluidics channels defined and TiN electrode as well as chambers where heater are present. Fig 9b shows the profile of polymer adhesive, the thickness achieved is roughly $30 \mu \mathrm{m}$ and very straight profile with a slight kink at the foot of the layer. Fig 9c shows a zoom out images along with heating chamber as well as sorting channels. Fig 9d shows the SEM images of the heating element in the chamber, which tungsten heater embedded in the passivation.

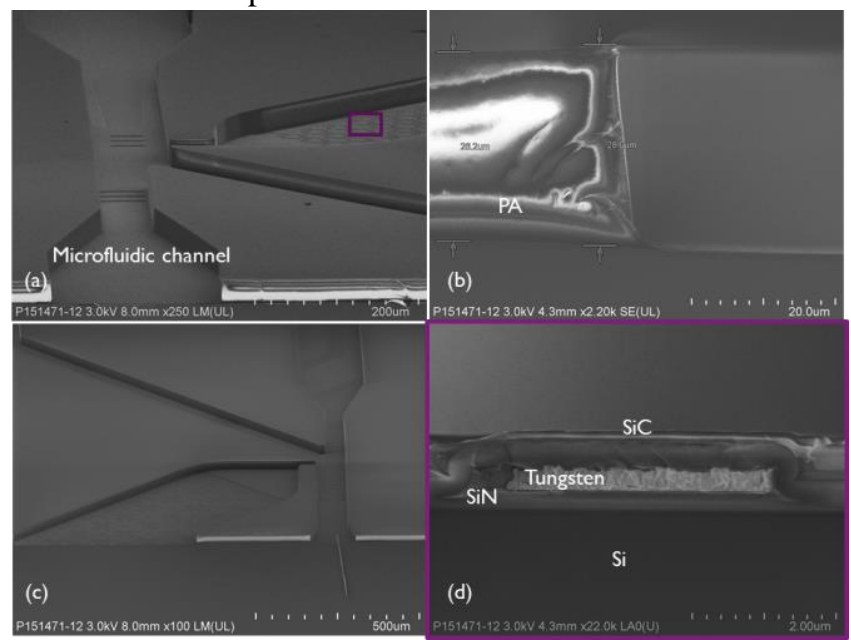

Figure 9:(a) Polymer adhesive patterned on device wafer (b) Polymer microfluidics channel with the $30 \mu \mathrm{m}$ height (c)zoom out image showing different component of cell sorter, (d) heating element with tungsten embedded in passivation

\section{Bonding results:}

After application of the polymer adhesive material, the last step is the bonding of glass die onto the silicon wafer. The glass die is externally sourced and holes are made in the glass die allowing connection from outside pumps to the polymer microfluidics channels on chip. The bonding step consist of two step, first single glass dies are bonded onto Si with a pick and place tool. The alignment is done with holes on glass to marker on the Si chip. During the populating step, the bottom wafer is mounted on a carrier and the glass dies are remounted on a tape on the ring. The bottom chuck is heated to a temperature $100^{\circ} \mathrm{C}$, while top chip is not heated and roughly $500 \mathrm{gF}$ is applied for $2 \mathrm{sec}$. After populating the wafer with required number of glass die, final bonding is done on BESI 2200 EVO Bonder. The top and bottom chuck temperature setting are the same. The force and temperature are applied simultaneously to complete the process. A design of experiment was done to show that the best bonding conditions were $300 \mathrm{~N}$ force per die at $200^{\circ} \mathrm{C}$ for 30 mintures. The quality of the bonding was checked with microfluidics test. At lower bonding force leakage between channels was observed, while at higher pressure the wafer broke. Fig. 10a shows the fully populated wafer with 10 dies on the wafer. Fig $9 b$ shows the glass dies with the 9 holes used for bringing in and taking out the sample to the die.

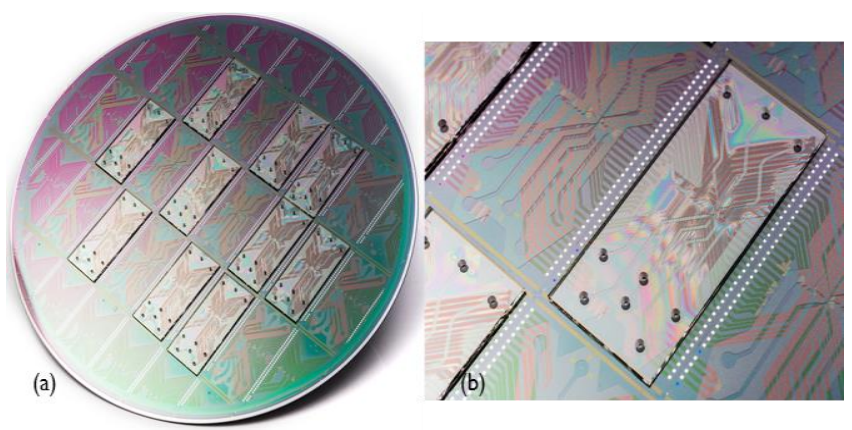

Figure 10: (a) 200mm device wafer with 10 dies populated (b) zoom image of singe die with holes in glass

\section{Characterization}

To characterize the device performance for sorting, we first measured the excitation condition for micro vapor bubble generation. A $20 \mu$ s wide, $0.16 \mathrm{~A}$ high current pulse was sent to each hotspot for vapor nucleation. With this excitation condition, totally 256 bubbles emerged in the heater micro chamber and produced a jet flow that sorted the flowing cells in the main fluidic channel.

Since the hotspot was very small and the current pulse was very short, the temperature is highly heterogeneous in space and time. It was not straightforward to measure the temperature. Therefore, we performed finite element thermal simulation in COMSOL to estimate the nucleation temperature. According to the early simulation, the hotspot temperature rose from room temperature to maximum $429 \mathrm{~K}$ in the $\mathrm{W}$ heater and $425 \mathrm{~K}$ in the $\mathrm{SiC}$-water interface as shown in Fig 11. The nucleation temperature was estimated between $388-413 \mathrm{~K} \quad\left(115-140^{\circ} \mathrm{C}\right)$. As expected, the nucleation temperature was higher than bulk nucleation temperature of water because of the superheat needed for fast bubble nucleation at smooth surface.

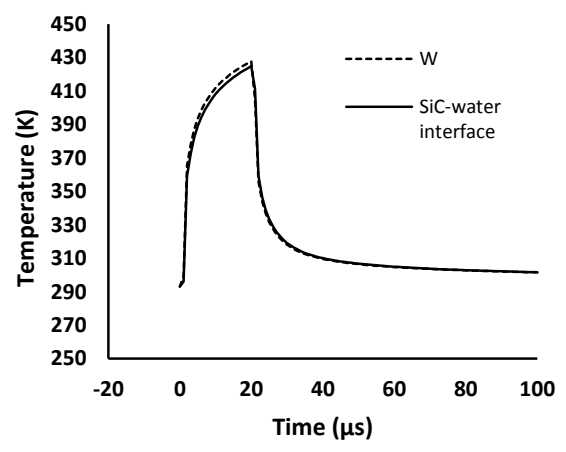

Figure 11: Heater temperature as function of time 
Noting that the current density in the tungsten heater was as high as $1.9 \times 10^{7} \mathrm{~A} / \mathrm{cm}^{2}$, already close to the electromigration limit [8], we tested the heater lifetime at 2 $\mathrm{KHz}$ without water. The tungsten heater was still intact after 0.5 billion firings. This is more than enough for most disposable cell sorting applications. Therefore, electromigration does not appear a major concern for the device lifetime with the improvement implemented in our current cell sorter device.

Lastly, the device was tested for actual sorting of fluorescent beads for demonstration. Figure 12 shows two different screen shots for the sorting applications. Fig. 12a shows the default situation where fluorescent beads are just passing thought the channels, when triggered, a jet flow is generated that sort the beads into the bottom channel as shown in Fig 12b. Once the jet flow is stopped the beads start moving in the central channel again. In the next stage of characterization, we looked at the sorting speed and yield of the sorted cell. From our initial test, we have achieved sorting rate of 5,000 cells/s and yield of $70 \%$.

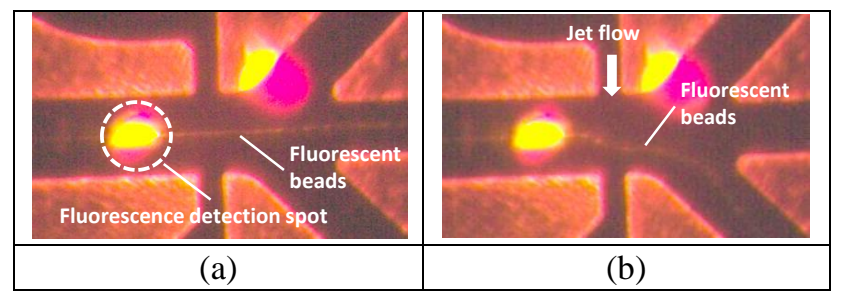

Figure 12. Fluorescent beads (a) not sorted when the jet flow is off, (b) sorted by jet flow.

\section{Conclusions}

Cell sorting is an important tool for molecular diagnostics where cell are sorted based on size and morphology. We have presented a solution that allows to miniaturize the cell sorting setup to a size of microscope slide. A CMOS compatible process was presented to develop heating element and integrate the chip with microfluidic component. The basic principle of our sorting device was the generation of heat pulse on a heater element that will create a bubble jet flow enabling the sorting. A reliable heating element is very important and we have shown in initial testing the heater to be reliable even after 500 million firings. The basic sorting mechanism with beads was presented and we have achieved very high through put sorting. In the next phase will be try to implement a wafer level bonding scheme that will increase the production volume and even allow for low volume manufacturing of the device.

\section{Acknowledgment}

The author thanks the European Research Council support under the Consolidator Grant (SCALPEL 617312 ). The authors will like to acknowledge the help of NCI colleague specially Tania Dupont and Evi Vrancken and for her help with cross-SEM analysis. Marc and Sofie for development of dry etching and special thanks to Caroline for all the help with the bonding experiments. Special thanks for JSR for providing the polymer material.

\section{References}

[1] J. Picot, C. L. Guerin, C. Le Van Kim, C. M. Boulanger, "Flow cytometry: retrospective, fundamentals and recent instrumentation," Cytotechnology, Vol 64, Iss. 2, Mar 2012, pp. 109-130

[2] H.R. Hulett, W.A. Bonner, J. Barrett, L.A. Herzenberg, "Cell sorting: automated separation of mammalian cells as a function of intracellular fluorescence," Science.; Vol. 166, 1969 Nov 7, pp.7479.

[3] IMEC On-Chip Single Cell Sorter [online] http://www.cytofluidix.com/imec-on-chip-single-cell-sorter/

[4] L. Lagae, D. Vercruysse, A. Dusa, C. Liu, K. de Wijs, R. Stahl, G. Vanmeerbeeck, B. Majeed, Y. Li, and P. Peumans, "High throughput cell sorter based on lensfree imaging of cells," In Proce .IEEE International Electron Devices Meeting - IEDM, Date 7-9 Dec. 2015, pp.13.3.1 - 13.3.4

[5] C. Liu, K de Wijs, D. Vercruysse, B Majeed, A Dusa, T. Miyazaki, P. Deshpande and L. Lagae "A high speed microfluidic fluorescent cell sorter by micro thermal jet flow," CYTO 2014, Fort Lauderdale, USA (17-21 May 2014).

[6] B. Majeed, C. Liu, L. Van Acker, R. Daily, T. Miyazaki, S. D. Tezcan, and L. Lagae, "Fabrication of silicon based microfluidics device for cell sorting applications," In proc. IEEE $64^{\text {th }}$ Electronic Components and Technology Conference ECTC. 2014, Orlando, USA. pp. $165-169$

[7] J. A. Maiz, "Characterization of electromigration under bidirectional (BC) and pulsed unidirectional (PDC) currents," Reliability Physics Symposium, 1989. 27th Annual Proce International, Phoenix, AZ, 1989, pp. 220-228.

[8] C. Martin and J. W. McPherson, "Via electromigration performance of ti/w/al-cu(2\%) multilayered metallization," VMIC Conference, pp. 168,1989

[9] J. A. Feinn and W R. Knight, "Non-kogating, low turn on energy thin film structure for very low Drop volume thermal ink jet pens" U.S. Patent 6,126,277 Oct. 3, 2000.

[10] D. W. Schulte, M. Giere, T. Sims, N.C. Lassar, M. Kent, "Thermal ink-jet print system and thermal ink-jet print head," Hewlett Packard co, JP20000251825, Mar 27, 2001

[11] JSR press release [online] http://www.jsrmicro.be/news/novelmicrofluid-material-breakthrough-wafer-scale-mass-production-labchip

[12] H. Kubo, T. Doi, I. Nishimura, E. Hayashi, K. Hieda, S. Peters and R. Vanroosbroeck, "Improvement of post exposure delay of photopatternable and adhesive materials for wafer-scale microfluidics," $J$. Photopoly. Sci. Tech. Vol. 28, 2015 No. 3, pp. 411-414. 